\title{
RESPONSABILIDAD SOCIAL DESDE EL PARADIGMA DEL DESARROLLO HUMANO EN LAS ORGANIZACIONES NO GUBERNAMENTALES
}

SOCIAL RESPONSIBILITY FROM THE HUMAN DEVELOPMENT PARADIGM IN NON-GOVERNMENTAL ORGANIZATIONS

\section{JESÚS RAMÓN MELÉNDEZ RANGEL', FRANCY LISSETH MONTOYA GÁMEZ²}

1 Universidad Católica de Santiago de Guayaquil. jesus.melendez@cu.ucsg.edu.ec

2 Universidad Centroccidental Lisandro Alvarado, Venezuela.francy.montoya@ucla.edu.ve

RESUMEN

La Responsabilidad Social (RS) es considerada un instrumento operativo que contribuye al mejoramiento del trabajador en sus dimensiones sociales, económicas y al mismo tiempo en su espacio-entorno empresarial. Sus postulados están orientados hacia el paradigma del Desarrollo Humano (DH); el cual sirve de referencia como incentivo hacia nuevos principios sobre la humanización y la valoración de las personas. El DH reivindica que la verdadera riqueza de las sociedades está en su gente (PNUD, 2002). Retribuyendo el valor intrínseco a la vida y a la expansión de la calidad de vida que merece todo ser humano. El presente artículo identifica los distintos aportes teóricos realizados por otros investigadores en el área gerencial de las Organizaciones No Gubernamentales (ONG) y de la RS. La metodología empleada, incluyó una revisión amplia de la literatura publicada en bases de datos y textos, considerando los temas relevantes y en contexto global. Se desarrollaron tres unidades de convergencias 0 categorías, para describir los contenidos que involucran a la RS, al DH y a las ONG. Finalmente, la discusión conduce a conocer la actualidad del tema. Se concluye que la RS implementada por las ONG está llenando los espacios que otras organizaciones del sector público y privado, aún no terminan de consolidar en materia del $\mathrm{DH}$.

PALABRAS CLAVE: responsabilidad social, organizaciones no gubernamentales, gerencia, desarrollo humano.
Currently, social responsibility (SR) has been considered the operative instrument that contributes to the improvement of the worker in its social and economic dimensions and at the same time in its space-environment, from the Human Development paradigm (HD). This article identifies the different theoretical contributions made by other researchers, in the management area of non-Governmental Organizations (NGO) and SR. The methodology used involved a broad review of the literature published in databases and texts, considering the relevant issues and in a global context. Three convergence units or categories were developed to describe the content of SR, HD and NGO. Finally the discussion leads to know the current topic. It is concluded that the SR implemented by non-governmental companies, is filling the spaces that other organizations of the public and private sector have not yet consolidated in HD.

KEYWORDS: social responsibility, non-governmental organizations, management, human development. 
INTRODUCCIÓN

En el mundo globalizado es común hablar de RS, una herramienta gerencial, aplicada por entidades públicas, empresas privadas y ONC que pertenecen al Tercer Sector. Todas ellas han vinculado algunos de los postulados del paradigma del DH para realizar sus planificaciones con el fin de ofrecer bienestar colectivo, a partir del diseño de sus políticas, planes, proyectos y programas. Es desde la segunda mitad del siglo pasado, específicamente en Norteamérica que la concepción sobre la RS se orientó hacia la gestión empresarial porque más allá de una obligación para alcanzar el crecimiento económico, era fundamental estar acompañada por un compromiso moral y ético del empresario (Melling \& Jensen, 2002). Este postulado aún se mantiene en la conceptualización moderna porque la esencia gerencial está fundamentada en la necesidad y la obligación de que toda organización que realiza cualquier actividad económica debe mantener una conducta ética y moral que esté relacionada al cumplimiento de las legislaciones laborales elementales y a la producción de bienes y servicios que suplan las necesidades de las comunidades (Bull, 2004).

En América Latina, la RS se considera un instrumento para la generación de bienestar. Constituye un elemento importante en los procesos de gestión de las organizaciones (Fernández y Velasco, 2013). Ha incorporado dimensiones del paradigma del DH establecidos en el concepto que maneja el PNUD (2002). Asimismo, las empresas transnacionales y locales adoptan dentro de sus estructuras elementos relacionados con la expansión de oportunidades y capacidades en sus públicos internos y externos, que permite observar un enfoque integral de fortalecer sus competencias profesionales e individuales (Fernández, 2011). No obstante, en este escenario, las ONG están considerando la adopción de las herramientas de la RS con el fin de fomentar mejoras en la calidad de vida de sus beneficiarios. En este sentido, se ha observado que una mala gestión en los procesos inherentes al desarrollo de los empleados, influye en el comportamiento organizacional desde el ámbito del rendimiento o la productividad.

En este marco de referencia, nos podemos preguntar, ¿Cuál es el papel que juega el paradigma del DH en la implementación de la RS en las ONG? Por lo tanto, el objetivo de este artículo es presentar varias posturas conceptuales sobre la RS y su vinculación con el paradigma del $\mathrm{DH}$ a través de la gestión que desarrollan las ONG. En este sentido, desde la perspectiva teórica y analítica, se realiza una revisión documental específica, la cual consiste en involucrar los distintos aportes teóricos de autores sobre el paradigma del DH, las ONG y la RS. La metodología de recolección de la información y los resultados obtenidos van a permitir conocer la influencia del DH en la aplicación de la RS en ONG con el propósito de mejorar el contexto integral de la calidad de vida del trabajador.

\section{METODOLOGÍA}

El artículo consideró como metodología implementar una revisión crítica y un análisis descriptivo de la literatura más relevante sobre investigaciones culminadas de varios autores en esta área de interés social. Se evaluaron los argumentos y se presentó una narración en secuencia de las aportaciones en el área de RS desde el paradigma del DH. Se consultaron bases de datos de journal de alto impacto y literatura internacional especializada. De esta manera se garantizó que las referencias citadas cumplan con las pautas de publicación académica.

CONCEPTUALIZANDO LA RESPONSABILIDAD SOCIAL

El tema de la RS, probablemente, ha sido el más abordado en los estudios realizados y discursos que se han pronunciado en el ámbito empresarial durante los últimos años. Sin embargo, existen diversas teorías con respecto a los antecedentes de la RS.

Los orígenes se sitúan en los Estados Unidos a finales de la década de los años cincuenta e inicios de los años sesenta del siglo pasado, como resultado de múltiples razones, entre las que se encuentran la presión de organizaciones sociales, comunidades y consumidores sobre aquellas empresas que adelantan prácticas irresponsables, relacionadas con la calidad de los productos y precios justos, sin especulación en los precios para los clientes, en el plano nacional e internacional.

En cuanto a las razones que propiciaron el surgimiento, diversos autores convergen con Alfaya (s. f.) cuando afirma que la RS surgió debido a los constantes cambios en el entorno socioeconómico mundial, ya que el proceso de globalización de la economía se ha acelerado en la última década como consecuencia del impresionante desarrollo de los medios de comunicación y del transporte de personas y bienes. De manera que, las decisiones adoptadas en las sedes corporativas de una multinacional pueden afectar a la economía de países enteros. Ante 
ello, se plantean nuevos retos a las empresas y es natural que cada vez, con más intensidad, la sociedad dirija sus exigencias y expectativas no sólo a los gobiernos, sino también a las grandes corporaciones empresariales.

La RS pretende la transformación del rol de las empresas, donde su función orientada estrictamente a la rentabilidad económica y maximizar el proceso financiero (Wang \& Sarkis, 2017). El objetivo se ha ampliado para incluir su finalidad como institución social. Según Guédez (2010) “(...) el concepto fue elaborado para actores empresariales, porque es, a ese tipo de actores, a los cuales las sociedades hacen una exhortación concreta a ir más allá de su función exclusivamente económica (...)” (p. 18).

Es por ello que Alfaya (s. f.) señala que a través de la RS las empresas pueden contribuir a crear un marco donde las mismas puedan gestionar sus operaciones de modo que se fomente el crecimiento económico y la competitividad; al tiempo que se garantice la protección del medio ambiente y se ponga en valor el impacto social de la actividad empresarial.

Las empresas, por su parte, comenzaron también a percatarse de que la puesta en marcha de políticas y acciones de RS constituían una fuente de beneficios en términos de reputación e imagen corporativa. Lo cual, a su vez, reduce el riesgo de la oposición pública contra sus operaciones, ayuda a conservar a los clientes y facilita el ingreso a nuevos mercados con mayores exigencias en términos de reducción de impactos negativos sobre el ambiente y la comunidad. Respecto a ello Alfaya (s. f.) señala que,

(...) cada vez son más los empresarios que ven en un comportamiento responsable un $\mathrm{ca}^{-}$ mino cierto para alcanzar el éxito comercial y proporcionar beneficios duraderos para sus accionistas. De ahí que algunas de las empresas líderes en todo el mundo hayan apostado firmemente por este camino como garantía de su supervivencia a largo plazo. (p. 11).

Sin embargo, aún no existe una definición totalmente aceptada sobre el significado de la RS debido a que el campo es relativamente nuevo y la proliferación bibliográfica ha dado lugar a una multiplicidad de conceptos vinculados a ella. Según la Red Iberoamericana de Universidades el concepto, es una forma de gestión que se define por la relación ética de la empresa con todos los públicos con los cuales ella se relaciona, y por el establecimiento de metas empresariales compatibles con el desarrollo sustentable de la sociedad; preservando recursos ambientales y culturales para las generaciones futuras, respetando la diversidad y promoviendo la reducción de las desigualdades sociales.

Sin embargo, Guédez (2010) obliga a pensar forzosamente en la RS como la humanización del capitalismo, sin llegar a poner en peligro la capacidad del sistema para producir bienes y servicios en forma masiva, ni reducir la competitividad, rentabilidad o capacidad de emplear.

En otro sentido, las empresas son responsables de los cambios que se producen en las sociedades y en el ambiente, debido a la toma de decisiones que su actividad productiva genera. En este contexto, deben tomar en consideración a sus socios o stakeholders, haciendo posible que se cumplan las leyes vigentes en materia de RS y de esta manera armonizar con la sociedad (Moreno y Graterol, 2010).

\section{RESPONSABILIDAD SOCIAL Y LAS ORGANIZACIONES NO GUBERNAMENTALES}

Al principio, la RS se concebía como una actividad meramente empresarial. En los años 90 del siglo pasado en las grandes corporaciones era asumida en diferentes contextos, especialmente en los relacionados con la política y la economía mundial. No obstante, para algunos autores, la raíz de la RS está vinculada con diversas concepciones acerca de la gestión empresarial que se efectuaba en Estados Unidos en los años 50 y 60, cuando era considerada una obligación moral y una responsabilidad del empresario, criterio que aún se mantiene (Díaz, 2014).

La aplicación de la RS ya a finales del siglo pasado, y a tono con los criterios emergentes de desarrollo, se da a partir de iniciativas establecidas en la implementación de normas y códigos orientados a lograr comportamientos éticos para con la sociedad y el ambiente. Ello, con el fin de construir un desarrollo sostenible, ya que su esencia gerencial está concebida en la necesidad y la obligación de las empresas que efectúan cualquier actividad económica, de mantener una conducta ética y moral.

Al calor del debate del desarrollo, la noción de RS se va ampliando, se incorpora más tarde a las organizaciones del Tercer Sector, específicamente en las ONG con el fin de presentar posturas responsables con la sociedad. Ser capaz de valorar aspectos como la calidad de vida, la protección de recursos naturales, dar beneficio a la comunidad, comercializar y mercadear 
con ética empresarial, entre otros criterios normativos inherentes a una gestión realmente responsable. Se trata entonces, de un conjunto de prácticas innovadoras dentro del sistema de gestión empresarial, a partir de un equilibrio entre las dimensiones económicas, sociales y ambientales, incluyendo el compromiso de transparencia y sobre todo, entendiendo que el ser humano es un ente integral, y que es parte de un sistema complejo y bio- psicosocial del cual forma parte la misma empresa (Guédez, 2010).

La aplicación de la gestión de la RS ha evolucionado en las últimas décadas como consecuencia, entre otros aspectos, de las demandas de la sociedad civil a través de las ONG; pero también como producto de reflexiones filosóficas y debates políticos en el ámbito mundial. En este sentido, aún existen un concepto limitado al aplicar RS en las empresas (Abugre \& Nyuur, 2015). Según Guédez (2010) es en el siglo XXI, cuando se retoma el concepto inicial de los años 50; RS como principio para desarrollar acciones gerenciales, al indicar que el debate sobre su alcance y su definición está relacionado con la filantropía, la inversión social, la ciudadanía corporativa y la responsabilidad con el entorno.

La RS implica a las empresas pero también a las ONG; por ello, no se cuenta con una única definición a pesar de los elementos en común hallados en las teorías analizadas. Según lo apreciado por Argandoña (2002) se trata "no de una realidad física, sino una construcción social" (p.1). De esta manera, hace énfasis en la responsabilidad de ejecutar las acciones porque las empresas tienen un impacto y una responsabilidad para con la sociedad.

La aplicación de la RS a través de las estrategias gerenciales, se ve reflejada cuando algunas organizaciones suscribieron los diez principios del Pacto Global presentados por la Organización de Naciones Unidas en el año 2000, el cual constituía una tribuna y un ejercicio práctico de las empresas para comprometerse con la sostenibilidad y la ética, a partir de refrendar los derechos humanos, laborales, medioambientales y de anticorrupción. Líderes mundiales promueven el compromiso de las empresas con la sociedad. En este sentido, la solidaridad y las alianzas entre instituciones son recursos clave para dar sentido al concepto instrumental de RS el cual permite ganar reputación y prestigio organizacional (Su, Pan, \& Chen, 2017). Desde estos puntos de vista, ser socialmente responsables conlleva modificar una actitud, reorientar las prácticas, ser críticos para tener un proyecto humano de reciprocidad y justicia desde diversas instancias de la sociedad.

La RS debe convertirse en una exigencia y no en una práctica circunstancial. En algunas ocasiones, los gerentes de corporaciones públicas, privadas o del Tercer Sector se encargan de definir sus prácticas de asistencialismo con el nombre de RS, dejando pasar la oportunidad de crear procesos de encuentro para enfrentar los retos contemporáneos del desarrollo en un mundo cada vez más globalizado y complejo.

La RS debe formar parte de la filosofía de gestión en las organizaciones y debe estar sustentada en un código de ética que contribuya a mantener buenas prácticas de gobernabilidad para apoyar al Estado a cumplir con sus compromisos públicos, sin descartar los procesos internos de seguridad laboral que protejan al trabajador de los riegos ocupacionales y al mismo tiempo mantener controles en sus áreas financieras y administrativas, que permitan disminuir prácticas desviadas como la corrupción. En este sentido, el enfoque global permite al DH integrar los esfuerzos y promover resultados eficientes para la organización.

En ese contexto, el enfoque gerencial de la RS debería estar orientado a que las organizaciones entiendan que el impacto de su comportamiento y sus operaciones determinan su habilidad para operar efectivamente. Es allí que la RS, desde un punto de vista operacional, se expande adquiriendo mayor resonancia como estrategia para la competitividad en el mundo moderno; haciendo énfasis en qué tan ética es la forma cómo las organizaciones se mantienen sustentables con los impactos económicos, sociales y medioambientales a la hora de tomar las decisiones, y que esto permita asegurar la continuidad y su presencia significativa en el tiempo (Kliksberg, 2004).

Los gerentes de algunas ONC de buena parte del mundo, parece que ya están apostando por la gerencia de la RS a tono con el DH. Se concentran en saber cuáles son las necesidades de la gente, expresadas en la ampliación de sus opciones y en fortalecer sus capacidades, aumentando sus oportunidades de educación, de acceso a la salud y de seguridad alimentaria para generar alternativas para las personas (Kliksberg, 2004).

Debemos aclarar, que en sentido estricto, no estamos comparando a las empresas con las organizaciones no lucrativas. Se trata de dos sectores dedicados a diferentes actividades, tienen distintos orígenes, formas legales de proceder e 
intereses. Además, porque se trata en muchas ocasiones de organizaciones nuevas, profesionalizadas en diferentes grados y por lo general, con problemas usualmente no relacionados con el mundo empresarial.

Las ONG, de naturaleza voluntaria y con capacidad de autogobierno, desde el ángulo de nuestro enfoque temático teórico, de alguna manera implican en su filosofía puntos en común con el DH; y en ese sentido, se trata de la sostenibilidad como otro componente clave. Esta, constituye parte de los pilares de la RS a partir del uso eficiente de los recursos, principalmente aquellos no renovables y la rentabilidad; ambos aspectos relacionados con el desarrollo económico de las organizaciones.

En países como Ecuador, las organizaciones estratégicas como la EP Petroecuador desde el año 2013 está mostrando la promoción del DH, al considerar elementos propios de la RS, y han definido su objetivo empresarial en incrementar e incentivar la especialización del talento humano, como una estrategia de mejoramiento del colectivo interno de la organización. De igual manera, se han hecho efectivo programas de compensación social a los trabajadores. En este sentido, el Ministerio de Telecomunicaciones y Sociedad de la Información del Ecuador (2016) da a conocer que los procesos digitalizados son llevados a la comunidad para el mejoramiento de sus condiciones sociales. Por lo tanto, de acuerdo con Enrique, Freire y Villao (2013) estamos en presencia de aportes a la solidaridad digital para mejorar la accesibilidad a otros sectores, como la educación y salud.

En este sentido, otras empresas públicas en el área de la producción de petróleos y energía en Venezuela, continúan mejorando y actualizando sus procesos relacionados a las mejoras del individuo dentro de su entorno empresarial y de manera más amplia al ámbito familiar. Es allí donde la RS se conecta con las comunidades para integrarse en un círculo concéntrico de mejora al colectivo, actualmente se realizan inversiones para la construcción de infraestructura y servicios que elevan el nivel de vida de los sectores y comunidades (Meléndez, 2017).

Estas empresas de diferentes sectores productivos, consideran a la RS como medio para el incremento de las oportunidades de mejoras de la calidad de los empleados y trabajadores en general, desde una concepción integral que favorece a sus círculos familiares e incluye la protección del ambiente.

\section{CONOCIENDO SOBRE EL DESARROLLO HUMANO}

Uno de los hitos que marca el inicio del paradigma del DH es la difusión del concepto a partir de la aparición del Informe sobre Desarrollo $\mathrm{Hu}^{-}$ mano del Programa de Naciones Unidas para el Desarrollo (PNUD) en el año 1990. No obstante, existen registros que indican que los orígenes datan desde los años 70 del pasado siglo, cuando se comienza a presentar el enfoque de las necesidades básicas aplicadas a los procesos de desarrollo que incluía aspectos sociales como la participación y el agotamiento de los recursos naturales. Uno de los ejemplos está relacionado con la solicitud que realiza la Organización Internacional del Trabajo (1976), cuando afirma que las prioridades del desarrollo tenían que cambiarse a favor de la generación de empleo y la satisfacción de necesidades humanas básicas tales como: alimento, vivienda, ropa, educación y salud.

El enfoque del DH es presentado como una propuesta que surge de la crítica a las corrientes de ideas dominantes basadas netamente en el crecimiento económico, la acumulación de capital, el desarrollo de recursos humanos o necesidades básicas; siendo las personas un medio y no un fin de estos procesos. Otras reflexiones, críticas y debates sobre el paradigma emergente del DH se están realizando desde la mitad del siglo pasado. Organismos internacionales como el PNUD, relevantes académicos, movimientos sociales y ONG de todo el mundo han hecho sus aportes, para dar paso a la ruptura del modelo economicista y ofrecer otras dimensiones del bienestar del ser humano. Desde la perspectiva de Sen (2000) el DH va más allá del enfoque basado netamente en el crecimiento económico y la acumulación de capital. Su basamento está en torno a las vidas de las personas, y más específicamente, en el nivel de libertad. Es decir, que los seres humanos puedan ser libres y puedan vivir su vida con calidad, evitando enfermedades y poder tener acceso al conocimiento a través de una buena educación.

Es importante señalar las acciones de organismos internacionales como la UNICEF, la cual implementó propuestas de programas para que se protegiera a los pobres de la grave crisis y de los recortes en los servicios básicos. En otro ámbito, la idea del economista y consultor del PNUD, Haq (1995), para elaborar una medición del DH, a través del Índice de Desarrollo Humano (IDH) del PNUD, eran distintas a las que se empleaban hasta ese momento. Más allá del cálculo desde el crecimiento económico, 
se debían incluir las dimensiones de igualdad y libertad. En este orden de idea Sen, (2009), agrega que "... el desarrollo puede concebirse como un proceso de expansión de las libertades [...], el crecimiento del Producto Nacional Bruto o de las rentas personales puede ser un medio muy importante para expandir las libertades de las que disfrutan los miembros de la sociedad" (p. 19).

Según Sen (2009) el enfoque del DH abarca las instituciones, ya sean públicas o privadas, además del sistema democrático, el mercado, los medios de comunicación, las organizaciones políticas y sociales, las organizaciones ciudadanas. Todas son observadas desde la perspectiva holística y sistémica porque deben ser incorporadas a los principios de justicia social para que puedan servir al desarrollo, tal y como lo explica al expresar que “... los trascendentales poderes del mecanismo de mercado han de complementarse con la creación de oportunidades sociales básicas para conseguir la equidad y la justicia social” (p. 179).

En la culminación del siglo XX e inicios del XXI es cuando mayor vigencia tiene la construcción y consolidación de este paradigma alternativo del DH porque contribuye y representa un panorama contemporáneo del pensamiento social de la comunidad internacional. Organismos multilaterales se nutren del IDH del PNUD para abordar y sustentar sus diagnósticos y ofrecer propuestas de diferentes perspectivas en la elaboración de las políticas públicas con el fin de hacer un quiebre entre el modelo del Fondo Monetario Internacional o el Banco Mundial en materia de desarrollo de la naciones.

Desde el enfoque de las capacidades, el DH se va forjando cuando las personas alcanzan la mayor libertad posible y los objetivos personales planteados. En este sentido, la democracia y la buena gobernanza están unidas con este enfoque ya que son factores claves para favorecer la ampliación de las capacidades (Sen, 2000). Se sustenta en la gobernabilidad y la democracia en las cuales pueden participar instituciones justas y responsables que defiendan los derechos humanos y las libertades y que los seres humanos que sean tratados como medios y fines de los procesos.

En este sentido, el DH va en función de combatir las inequidades sociales a partir de sugerir la aplicación de procesos de formación para que las personas reconozcan y visualicen la vida que quieren llevar. Destaca el PNUD (2002), que el DH resulta ser una combinación de capacidades y oportunidades. Se hace hincapié en que las personas sean el centro del desarrollo, a partir de propiciar ambientes sociales garantes de oportunidades. Igualmente, considerar que el paradigma del DH es integral. Incluye aspectos sociales, económicos y ambientales, relacionados con aspectos históricos, de conducta humana y del sistema organizacional (Hernández y Escala, 2011).

\section{ORGANIZACIONES NO GUBERNAMENTALES}

El origen y el nombre de las ONG surgen después de la Segunda Guerra Mundial. Según Vallejo de la Pava (2001) las ONG son "organizaciones voluntarias de ciudadanos sin ánimo de lucro, nacional o internacional” (p. 66). Sin embargo, Montero (2003) la define como "organizaciones de naturaleza voluntaria y altruista y con capacidad de autogobierno que intervienen en el campo del bienestar social con el fin de mejorar las condiciones de calidad de vida de la sociedad en general o de un sector concreto de la misma" (p. 31). De esta manera, seinterpreta quelas ONC son agentes de acción social y que cuentan con el apoyo activo de los miembros de la sociedad civil con el objetivo de conquistar partidarios, llamar la atención de un cierto público y de las élites con el fin de lograr una posición mediadora entre los movimientos sociales y las instituciones con las que se relacionan, o a la inversa.

El World Bank (2004), considera que las ONG forman parte de una economía de mercado y son responsables del bienestar social. Entre sus funciones están: aliviar sufrimientos, promover los intereses de los pobres, proteger el ambiente, proveer servicios sociales fundamentales o fomentar el desarrollo comunitario. De esta manera, las ONG constituyen uno de los sectores sociales más heterogéneos y diversos que forman parte de una sociedad, tratando de aglutinar a varios actores de la población organizada para proceder de manera autónoma dentro de contextos políticos, económicos y culturales de poder. La denominación no gubernamental le vale para hacer hincapié en su independencia y le brinda legitimidad para participar en cualquier lugar del planeta, más allá de la ideología de los pueblos o de las personas.

La participación de las ONG en algunas ocasiones complementa las acciones que debería cumplir el Estado; el cual requiere contar con la colaboración de la sociedad organizada para cumplir con sus metas. Darle una mano al Estado no las hace organizaciones públicas; pero sí contribuyen a facilitar procesos de las políticas 
económicas gubernamentales a través de su participación en el debate democrático para el desarrollo de las comunidades. Por tal motivo, se convierten en agentes de cambio para mejorar la gestión, ampliar la participación local, promover la innovación y capacitar en la promoción del uso de la tecnología a través de la implementación de proyectos de poca inversión.

Es oportuno destacar que, en pleno siglo XXI es necesario desmitificar la labor que desarrollan las ONG de su condición de asistencialismo, filantropía y pasividad que tenían en su origen. Ahora hay que afianzar que no forman parte del círculo existente entre el aparato del Estado, los sindicatos y las asociaciones. Sus objetivos tienen su génesis fuera del lucro y su metodología está orientada a romper con la pasividad de las personas a partir de promover sentido de conciencia.

En este orden de ideas, se puede establecer que una de las características que agrupa a las ONG, es la posibilidad de ampliar las $\mathrm{ca}^{-}$ pacidades de las personas para que alcancen la felicidad de manera individual y también colectivamente; todo a través de un proceso de asesoramiento (Montero, 2003). No existe una definición única de las ONG, hecho que ha contribuido a generar nuevos espacios para la reflexión. En el escenario social, se han agrupando múltiples iniciativas heterogéneas, tales como las cooperativas, asociaciones, fundaciones, federaciones, consejos, comité y sociedades, entre otras, que proyectan sus actividades sin ánimo de lucro por encima de la frontera del Estado.

A la mitad del siglo pasado, las ONG inician una nueva etapa, cuando surge Amnistía Internacional, organismo internacional que tiene como misión defender los derechos humanos y el Fondo Mundial de la Vida Salvaje (WWF por sus iniciales en inglés) encauzado a la protección del ambiente (Vallejo de la Pava, 2001). Más adelante, aparece Human Rights Watch, Greenpeace y Médicos Sin Fronteras; todas estas ONG cuentan con relevancia y transcendencia en el ámbito mundial. Estas organizaciones ampliaron su radio de acción pasando de tener influencia en las políticas públicas estatales, a intervenir en los procesos de toma de decisiones en organismos intergubernamentales como lo hace la Organización Mundial de la Salud o la Organización Mundial del Comercio.

En las últimas décadas, algunas ONC han dirigido su atención a temas relacionados con las prácticas de la RS. Este hecho se ve respal- dado a través de las diferentes acciones que se observan en la agenda mundial como lo es el Pacto Clobal, los Objetivos de la Declaración del Milenio del año 2000 y recientemente los Objetivos de Desarrollo Sostenible 2015/2030. Orientados todos a promover la calidad en función de la sostenibilidad (Guerra, 2014). Lo antes expuesto, se encuentra en concordancia con la postura de Sen (2009) quien considera que el proceso de expansión de las libertades reales de que disfrutan los individuos, constituye el mejoramiento de sus capacidades generales a través de la transparencia y la seguridad.

\section{DISCUSIÓN}

Las investigaciones consideradas para desarrollar este artículo, estuvieron dentro de un contexto que da a conocer a la RS, como un instrumento que promueve una ética ciudadana y empresarial e impulsa que el DH sea justo y sustentable desde las ONG.

Se trata de una versión para innovar la concepción que se tenía de desarrollo desde la visión economicista y apuntar hacia tener y propiciar la construcción de comunidades con sentido de pertenencia, identidad, vínculo y solidaridad entre las personas.

Asimismo, podemos resaltar que las ONG están cumpliendo en Latinoamérica y en el mundo globalizado un rol fundamental en la transformación de la gerencia moderna, dando respuestas a partir de las inquietudes de una sociedad organizada y en pleno proceso de globalización. Hoy más que nunca es prioridad incluir herramientas de RS en los procesos de una gestión sustentable, participativa, equitativa y responsable de las ONG.

En las sociedades latinoamericanas se concreta un auge de la seguridad social complementaria como elemento integrador del concepto amplio de protección social de los trabajadores; ubicada en el grupo de normas constitucionales encargadas de asegurar un status jurídico material del ciudadano en el cual se le conceda un sentido amplio a la seguridad social (Martínez, 2006).

\section{CONCLUSIONES}

Los datos aportados por el IDH en los últimos años, reflejan un considerable avance del $\mathrm{DH}$, incluso en países poco industrializados como los de América Latina. A ello, se suma la incorporación de nuevas herramientas gerenciales como la RS por parte de las ONG al momento de formular sus estrategias que tienen entre su 
misión, cumplir con los principios de democracia, equidad y sustentabilidad, pilares todos del paradigma del $\mathrm{DH}$.

Son los gerentes, quienes tienen en su poder considerar las competencias y los conocimientos individuales de los profesionales, a través de sus capacidades y facultades para desarrollar programas acertados que permitan llegar a la meta de la organización. Contar con el personal idóneo para lograr los objetivos siempre es uno de los recursos más valiosos. En este orden de ideas, los gerentes de las organizaciones de buena parte del mundo, ya están apostando a conjugar y hacer realidad los retos para alcanzar el DH a través de la aplicación de la gestión de la RS. En este proceso, se concretan cuáles son las necesidades de las personas, expresadas en la ampliación de sus opciones y de fortalecer sus capacidades, aumentando sus oportunidades de educación, de acceso a la salud y de seguridad alimentaria, y se consideran alternativas para valorar el tipo de vida que desean (Kliksberg, 2004). Conseguir un mundo cada vez más equitativo en lo económico, social, político y cultural es uno de los retos que debe tomar en consideración la gerencia moderna.

Igualmente, es importante disminuir la brecha entre países desarrollados y en vías de desarrollo a través de la democratización de la innovación tecnológica; además de socializar y globalizar el conocimiento, y la articulación entre mercado, Estado y sociedad civil para el incremento de la productividad con criterios sustentables. En este sentido, Crespo, López, Peña y Carreño (2003), expresan que la seguridad social se refiere a un conjunto de medidas asumidas por el Estado que tienen por objeto la protección, ayuda y remedio a los ciudadanos en determinadas situaciones de riesgo, desamparo o siniestro; así como la prevención de las mismas, contribuyendo a mejorar la situación social del trabajador de las organizaciones.

Las ONG son consideradas un motor importante en la praxis del DH. Han incorporado las dimensiones de la RS a través de sus estrategias ampliando las oportunidades de acceso a sus trabajadores, comunidades externas y sociedad en general. La opción de ser autónomas e independientes del sector público y del privado ha contribuido a que sus funciones de participación, y en algunas ocasiones de empoderamiento de la población, se vea reflejada en las áreas de la política, la economía y la cultura.

Se concluye que las ONG promueven, constituyen y afianzan el trabajo de la sociedad organizada; ya que en muchas ocasiones ofrecen servicios de salud antes o primero que las instituciones gubernamentales. Se han convertido en una esperanza de los sectores más vulnerables, los cuales han logrado organizarse de manera voluntaria, contando con una extraordinaria capacidad de ampliar su propio desarrollo porque han logrado multiplicar la capacidad de los individuos aislados y sin poder de decisión para ser protagonistas de su DH.

Finalmente, el tema de la RS y sus implicaciones desde el paradigma del $\mathrm{DH}$, es complejo y continúa siendo revisado por otros investigadores de las áreas sociales; por lo que sugerimos continuar investigando y profundizando en esta dirección.

\section{REFERENCIAS BIBLIOGRÁFICAS}

Abugre, J. B., \& Nyuur, R. B. (2015). Organizations' commitment to andcommunication of CSR activities: insights from Ghana. Social ResponsibilityJournal, 11(1), 161-178. Recuperado de https://doi.org/10.1108/SRJ-06-2013-0066.

Alfaya, V. (s. f.). La responsabilidad social empresarial. La empresa Sostenible. Madrid: Ferrovial.

Argandoña, A. (2002). Otra definición de Responsabilidad Social. Recuperado de http://www.iese.edu/en/ files/catedra\%20abril_tcm4-79450.pdf

Bull, B. (2004). Responsabilidad social de las empresas: ¿Una solución para el Desarrollo en América Latina? Revista Venezolana de Gerencia, 9, 1- 24. Recuperado de http://sociales.redalyc. org/articulo.oa?id=29092802

Crespo, T., López, J., Peña, J.E., y Carreño, F. (2003). Administración de Empresas. Madrid, España: Mad.

Díaz, C. N. (2014).Responsabilidad Social Empresarial y Creación de Valor Compartido, Sostenibilidad Gerencial. Revista Daena (International Journal of Good Conscience), 9(3). Recuperado de http://www.spentamexico.org/v9-n3/ A10.9(3)127-144.pdf

Enrique, E., Freire, G., y Villao, F. (2013). Estructurar una propuesta para el Plan nacional de desarrollo de las telecomunicaciones 2013-2017. Recuperado de https://www.dspace.espol.edu. ec/bitstream/123456789/25374/1/Resumen\%20 de $\% 20$ Tesis $\% 20$ EErique $\% 20 y \% 20$ GFreire, $\% 20$ director $\% 20$ de $\% 20$ tesis $\% 20$ Ph.D. \%20Freddy $\% 20$ Villao\%2021\%20nov\%202013.pdf

Fernández, I. A., y Velasco, C. Á. B. (2013). Responsabilidad social y ética. Aportaciones en el ámbito de los estudios sobre responsabilidad Social. Boletín de EstudiosEconómicos, 68(208), 165- 
181. Recuperado de https://search.proquest. com/docview/1364761135?accountid=38660

Fernández, S. (2011). Política social y Desarrollo Humano.La nueva cuestión social del siglo XXI. Nómadas, 29(1), 1-21. Recuperado dehttps://search.proquest.com/docview/9409362 53?accountid $=38660$

Guédez, V. (2010). Ser confiable. Responsabilidad social y reputación Empresarial. Caracas, Venezuela: Editorial Planeta.

Guerra, A. (2014). Desarrollo Humano Integral: Estudio introductorio. En A. Guerra, y C. Giménez (comps), Desarrollo Humano Integral compromiso de todos (pp.17-83). Caracas, Venezuela: Ediciones Del Banco Central de Venezuela y Cátedras Libres de la UCLA. Recuperado de https://issuu.com/edicionesmoon/docs/desarrollo_humano_integral_compromi

Haq, M. (1995). El Paradigma Del Desarrollo Humano. Recuperado de http://www.otrodesarrollo. com/desarrollohumano/MulHaqParadigmaDesarrolloHumano.pdf

Hernández, A., y Escala, Z. (2011). Enfoque de las Capacidades y el Desarrollo Humano, origen, evolución y aplicaciones. Caracas, Venezuela: Total Oil \& PNUD.

Kliksberg, B. (2004). La ética y el capital social cuentan. Caracas, Venezuela: Ediciones de la Facultad de Ciencias Económicas y Sociales de la Universidad de Carabobo.

Martínez, M. (2006). Relaciones entre empleo y seguridad social. Barcelona, España: Netbiblo.

Meléndez, J.R. (2017). Administración y gestión de riesgos en proyectos de la Empresa Pública. Guayaquil, Ecuador: Dirección de Publicaciones de la Universidad Católica de Santiago de Guayaquil.

Melling, C., \& Jensen, S. (2002). From Charity to Necessity. An Introduction to The Concept of Corporate Social Responsibility. Report $\mathrm{N}^{\circ}$ 2. Trondheim: Department of Cross- Cultural Studies, Norway's Technical Scientific Nature of the Witness University (NTNU).

Ministerio de Telecomunicaciones y Sociedad de la Información. (2016). Plan nacional de telecomunicaciones y tecnologías de información del Ecuador 2016-2021. Recuperado de https:// www.telecomunicaciones.gob.ec/wp-content/ uploads/2016/08/Plan-de-Telecomunicacionesy-TI.
Montero, M. J. (2003). El marketing en las ONGD. La gestión del cambio social. Málaga, España: Editorial Desclée de Brouwer S.A.

Moreno, Z., y Graterol, D. (2010). Prácticas de responsabilidad social Empresarial en la Pyme. Un estudio en el sector metalmecánico de Barquisimeto, Estado Lara, Venezuela. Revista científica Contaduría y Administración UNAM, 235, 175-194. Recuperado de http://www.scielo. org.mx/pdf/cya/n235/n235a9.pdf

Organización Internacional del Trabajo. (1976). Convenio sobre consultas tripartitas para promover la aplicación de las normas internacionales del trabajo. Recuperado de http://www.ilo.org/dyn/normlex/ es/f?p=NORMLEXPUB:12100:0::NO ::P12100_INSTRUMENT_ID:312289

PNUD. (2002). Informe sobre Desarrollo Humano. Boletín de Estudios Económicos, 68(208), 165-181. Recuperado de http://www.undp.org/content/undp/es/home.html

Sen, A. K. (2000). Desarrollo económico como libertad. Revista Científica de Apuntes del CENES, (55), 14-20. Recuperado de https://dialnet.unirioja.es/servlet/ articulo?codigo $=2899993$

Sen, A. K. (2009). Desarrollo económico y libertad. Revista Científica de Apuntes del CENES, (48), 367 - 386. Recuperado de http://www.Dialnet-DesarrolloEconomicoYLibertad-3399366.pdf

Su, L., Pan, Y., \& Chen, X. (2017). Corporate social responsibility: Findings from the Chinese hospitality industry. Journal of Retailing and Consumer Services, 34, 240-247. Recuperado de https://doi.org/10.1016/j. jretconser.2016.10.013

Vallejo de la Pava, A. (2001). Organizaciones no gubernamentales: Supuestos, Evidencia y Capacidad. Revista civilizar, 1, 63-83. Recuperado de http://www. usergioarboleda.edu.co/civilizar

Wang, Z., \& Sarkis, J. (2017). Corporate social responsibility governance, outcomes, and financial performance. Journal of Cleaner Production, 162, 1607-1616. Recuperado de https:// doi.org/10.1016/j.jclepro.2017.06.142

World Bank. (2004). NGO World Bank collaboration. Recuperado de http://wbln0018.workbank.org/essd/essd.nsf/ d3f59aa3a570f67a852567cf00695688/ce6b105aaa19 360f85256966006c74e3?OpenDocument 